\title{
DENSELY DEFINED SELECTIONS OF MULTIVALUED MAPPINGS
}

\author{
M. M. ČOBAN, P. S. KENDEROV, AND J. P. REVALSKI
}

\begin{abstract}
Rather general suficient conditions are found for a given multivalued mapping $F: X \rightarrow Y$ to possess an upper semicontinuous and compactvalued selection $G$ which is defined on a dense $G_{\delta}$-subset of the domain of $F$. The case when the selection $G$ is single-valued (and continuous) is also investigated. The results are applied to prove some known as well as new results concerning generic differentiability of convex functions, Lavrentieff type theorem, generic well-posedness of optimization problems and generic nonmultivaluedness of metric projections and antiprojections.
\end{abstract}

\section{INTRODUCTION}

Let $F: X \rightarrow Y$ be a multivalued mapping with nonempty images acting from the topological space $X$ into the topological space $Y$ (i.e., for each $x \in X$ the value $F(x)$ is a nonempty subset of $Y$ ). We give here sufficient conditions for the existence of an upper semicontinuous and nonempty compact-valued (u.s.c.o.) mapping $G: X_{1} \rightarrow Y$, where $X_{1}$ is a dense $G_{\delta}$-subset of $X$ and $G$ is a selection of $F$. In contrast to what is widely accepted under "a selection of $F$ on $X_{1}$ "we understand here a set-valued mapping $G: X_{1} \rightarrow Y$ such that $\varnothing \neq G(x) \subset F(x)$ for every $x \in X_{1}$. In some particular cases the selection $G$ coincides with the restriction of $F$ on $X_{1}$. If the range space $Y$ is completely metrizable, then $G$ can be considered to be single-valued.

More precisely, we call $F$ lower demicontinuous (l.d.c.) in $X$ if for every open $V$ in $Y$, the interior of the closure of the set $F^{-1}(V):=\{x \in X: F(x) \cap$ $V \neq \varnothing\}$ is dense in the closure of $F^{-1}(V)$, i.e., $\operatorname{Int} \mathrm{Cl}\left(F^{-1}(V)\right)$ is dense in $\mathrm{Cl}\left(F^{-1}(V)\right)$. The consept of l.d.s. mappings will be discussed in more detail in $\S 4$. Several results are proved in $\S 4$ asserting that a given l.d.c. mapping $F$ admits an u.s.c.o. selection defined on a dense subset of $X$. A typical theorem (see Theorem 4.6 for full generality) reads as follows:

Let $F: X \rightarrow Y$ be a 1.d.c. mapping with closed graph, where $X$ is a Baire space and $Y$ is Čech complete. Then there exist a dense $G_{\delta}$-subset $X_{1}$ of $X$ and an u.s.c.o. mapping $G: X_{1} \rightarrow Y$ which is a selection of $F$. If, in addition, $Y$ is completely metrizable, then $G$ can be considered to be single-valued (see

Received by the editors September 11, 1991.

1991 Mathematics Subject Classification. Primary 54C65, 54C60, 46B20.

Key words and phrases. Multivalued mappings, selections, semicontinuity, generic continuity, Baire category.

This study was partially supported by the Bulgarian Ministry of Education and Science under contract No. 127. 
Theorem 4.7). In some cases the mapping $G$ can be constructed in such a way that its values belong to a subset $Y_{1}$ of $Y$ which is specified in advance.

Theorem 4.6 should be compared to the following theorem of E. Michael [M2] (although, the two results are different in nature): if the space $X$ is paracompact, $Y$ is a complete metric space and $F$ is lower semicontinuous and closed-valued, then an u.s.c.o. selection $H$ of $F$ exists which is defined on all of the space $X$.

Theorems 4.8 and 4.9 generalize some of the results in the paper of E. Michael [M4]. Our example 4.15 gives a negative answer to Question 7.4 from the same paper of Michael [M4].

In $\S 5$ we describe situations in which the mapping $F$ itself (not merely a selection of it) is u.s.c.o. at the points of a dense $G_{\delta}$-subset of $X$. A partial case of Theorem 5.2 says that, if $F: X \rightarrow Y$ has a closed graph, $X$ is a Baire space, $Y$ is Čech complete and for every open $V \subset Y$ the interior of the set $\{x \in X: F(x) \subset V\}$ is dense in $F^{-1}(V)$, then there exists a dense $G_{\delta}$-subset $X_{1}$ of $X$ at the points of which $F$ is u.s.c.o. If, in addition, $Y$ is completely metrizable then $F$ is single-valued at the points of $X_{1}$ (see Theorem 5.3). Theorem 5.3 is used to derive known results about generic nonmultivaluedness of metric projections and antiprojections (Theorem 6.10).

Theorem 5.4 is applied in $\S 6$ to prove a new version of the classical Lavrentieff theorem concerning the extension of a densely defined homeomorphism to a subset containing a dense $G_{\delta}$-subset of the domain space (Theorem 6.4). As shown in Theorem 6.5 the selection theorems could be used to prove that a given convex and continuous function is differentiable at the points of some dense $G_{\delta}$-subset of its domain.

Finally, Theorems 6.1 and 6.2 show that the main results from our previous papers [ČKK1, С̌K2, ČKR1, and ČKR2] are, in essence, easily derived from the results in $\S 4$.

The general construction used in the proofs of our results is described in Theorems 3.1 and 3.3.

\section{Preliminaries}

We recall briefly some notions and continuity properties of multivalued mappings. Let $F: X \rightarrow Y$, where $X$ and $Y$ are Hausdorff topological spaces, be such a mapping. By $\operatorname{Dom}(F)$, as usual, we denote the domain of $F$, i.e., the set $\{x \in X: F(x) \neq \varnothing\}$. If $A$ and $B$ are subsets of $X$ and $Y$ respectively, then by $F(A)$ we designate the set $\bigcup\{F(x): x \in A\}$ and by $F^{-1}(B)$ and $F^{\#}(B)$ the sets $\{x \in X: F(x) \cap B \neq \varnothing\}$ and $\{x \in X: F(x) \subset B\}$ correspondingly. In particular $F^{\#}(B)$ contains all points $x \in X$ for which $F(x)=\varnothing$. The set $\operatorname{Gr}(F):=\{(x, y) \in X \times Y: y \in F(x)\}$ denotes the graph of $F$. We will say that $F$ has a closed graph if $\operatorname{Gr}(F)$ is a closed subset of the product topology in $X \times Y . F$ is said to be upper (resp. lower) semicontinuous at a point $x_{0} \in X$ if for every open $V \subset Y$ such that $F\left(x_{0}\right) \subset V$ (resp. $F\left(x_{0}\right) \cap V \neq \varnothing$ ) the set $F^{\#}(V)$ (resp. the set $F^{-1}(V)$ ) contains an open neighborhood of $x_{0}$. In this case we will write that $F$ is u.s.c. (resp. l.s.c.) at $x_{0} . F$ is u.s.c. (resp. 1.s.c.) in $X$ if it is u.s.c. (resp. l.s.c.) at any point of $X . F$ is called u.s.c.o. if it is u.s.c. and compact-valued. Every u.s.c.o. mapping $F$ has a closed graph. 
Conversely, if the range space $Y$ is compact and $F$ has a closed graph then $F$ is u.s.c.o.

An u.s.c.o. $F$ is minimal if its graph does not contain properly the graph of any other u.s.c.o. mapping with the same domain. It can be seen (by the Kuratowski-Zorn lemma) that for every u.s.c.o. mapping $F: X \rightarrow Y$ there exists a minimal u.s.c.o. $G: X \rightarrow Y$ which is contained in $F$ and has the same domain. The next statement is well-known and summarizes what we will need about minimal u.s.c.o. mappings.

Proposition 2.1. The following are equivalent for an u.s.c.o. $F: X \rightarrow Y$ :

(a) $F$ is minimal;

(b) for every open $U$ in $X$ and closed $B$ in $Y$ from $F(x) \cap B \neq \varnothing$ for every $x \in U \cap \operatorname{Dom}(F)$, it follows that $F(U) \subset B$;

(c) if $U$ and $V$ are open subsets of $X$ and $Y$ such that $(U \times V) \cap \operatorname{Gr}(F) \neq \varnothing$ then there is a nonempty open $U^{\prime} \subset U$ with $U^{\prime} \cap \operatorname{Dom}(F) \neq \varnothing$ and $F\left(U^{\prime}\right) \subset V$.

In the sequel only Hausdorff topological spaces will be considered, although some of the results are true for spaces which are not necessarily Hausdorff. For a given subset $A$ of the topological space $Z$ we denote by $\mathrm{Cl}_{Z}(A)$ and $\operatorname{Int}_{Z}(A)$ the closure and the interior of $A$ in $Z$. If there is no danger of ambiguity we will omit the subscript $Z$. The completely regular space $Z$ is said to be $\check{C} e c h$ complete if it lies as a $G_{\delta}$-subset in its Stone-Cech compactification $\beta Z$ (or in any other compactification of $Z$ ).

Slightly modifying an idea from [Cᄃ̌N], under a sieve $\mathbf{s}$ in the space $Z$ we mean a sequence of indexed families of nonempty subsets $\left\{V_{\alpha}: \alpha \in A_{n}\right\}, n \geq 0$, of $Z$ and a sequence of single-valued mappings $\pi_{n}: A_{n+1} \rightarrow A_{n}$, such that $V_{\alpha}=$ $Z$ for $\alpha \in A_{0}$ and for every $\alpha \in A_{n}, n \geq 0, V_{\alpha} \supset \bigcup\left\{V_{\beta}: \beta \in \pi_{n}^{-1}(\alpha)\right\}$. Observe that we do not require $\left\{V_{\beta}: \beta \in \pi_{n}^{-1}(\alpha)\right\}$ to be a cover for $V_{\alpha}, \alpha \in A_{n}, n \geq 0$ (and hence $\left\{V_{\alpha}: \alpha \in A_{n}\right\}$ need not to be a cover for $Z$ ). We remark also that the sets $V_{\alpha}, \alpha \in A_{n}, n \geq 0$, need not be open. A sequence $\left\{\alpha_{n}\right\}, \alpha_{n} \in A_{n}$, for this sieve is called a $\pi$-chain if $\pi_{n}\left(\alpha_{n+1}\right)=\alpha_{n}$ for every $n \geq 0$. By $K(\mathbf{s})$ we denote the kernel of $\mathbf{s}$, i.e. the set $\left\{x \in Z: x \in \bigcap_{n=0}^{\infty} V_{\alpha_{n}}\right.$ for some $\pi$-chain $\left.\left\{\alpha_{n}\right\}\right\}$.

The sieve $\mathbf{s}$ is called complete if for each $\pi$-chain $\left\{\alpha_{n}\right\}$ the set $\bigcap_{n=0}^{\infty} V_{\alpha_{n}}$ is nonempty and compact and for every open $U$ in $Z$ such that $\bigcap_{n=0}^{\infty} V_{\alpha_{n}} \subset U$ there exists some $n$ with $V_{\alpha_{n}} \subset U$. The sieve $\mathbf{s}$ is open if every $V_{\alpha}$ is open.

\section{MAIN CONSTRUCTION AND BASIC RESUltS}

This section is devoted to the existence of an u.s.c.o. selection of a given multivalued mapping $F: X \rightarrow Y$ which is defined on a residual subset of the domain space $X$. Recall that a subset $A$ of the topological space $X$ is residual in $X$ if its complement $X \backslash A$ is of first Baire category in $X$, i.e., $X \backslash A=$ $\bigcup_{n=1}^{\infty} H_{n}$, where $\operatorname{Int} \mathrm{Cl}\left(H_{n}\right)=\varnothing$. Every dense $G_{\delta}$-subset of the space $X$ is residual in $X$. The space $X$ is said to be a Baire space if the intersection of every countable family of open and dense subsets of $X$ is dense in $X$. By a selection of $F$ on the subset $A$ of $\operatorname{Dom}(F)$, we mean a multivalued mapping $G: A \rightarrow Y$ such that $\varnothing \neq G(x) \subset F(x)$ for every $x \in A$. We start with the main result in this paper. 
Theorem 3.1. Let $F: X \rightarrow Y$ be a mapping with closed graph and such that $\operatorname{Dom}(F)$ is dense in $X$. Let $X$ be a Baire space and $Y$ admit a complete sieve $\mathbf{s}=\left(\left\{V_{\alpha}: \alpha \in A_{n}\right\},\left\{\pi_{n}\right\}\right)$ such that $\bigcup\left\{\operatorname{Int} C l F^{-1}\left(V_{\beta}\right): \beta \in \pi_{n}^{-1}(\alpha)\right\}$ is dense in Int $\mathrm{Cl}^{-1}\left(V_{\alpha}\right)$ for every $\alpha \in A_{n}$ and $n \geq 0$. Then there exist a dense $G_{\delta}$-subset $X_{1}$ of $X$ and an u.s.c.o. mapping $G: X_{1} \rightarrow K(\mathbf{s})$ from $X_{1}$ into the kernel of the sieve $\mathbf{s}$, such that:

(a) $X_{1} \subset \operatorname{Dom}(F)$;

(b) $G$ is a selection of $F$ on the set $X_{1}$.

Proof. Let us consider the complete sieve $\mathbf{s}=\left(\left\{V_{\alpha}: \alpha \in A_{n}\right\},\left\{\pi_{n}\right\}\right)$ in $Y$. Give the sets $A_{n}$ well-ordering in such a way that the mappings $\pi_{n}$ are monotone, i.e., if $\beta, \gamma \in A_{n+1}$ and $\gamma<\beta$ then $\pi_{n}(\gamma) \leq \pi_{n}(\beta)$. Put now for every $\alpha \in$ $A_{n}, n \geq 0, W_{\alpha}:=\operatorname{Int} \mathrm{Cl}^{-1}\left(V_{\alpha}\right), U_{\alpha}:=W_{\alpha} \backslash \mathrm{Cl}\left(\bigcup\left\{W_{\beta}: \beta \in A_{n}, \beta<\alpha\right\}\right)$ and let $B_{n}:=\left\{\alpha \in A_{n}: U_{\alpha} \neq \varnothing\right\}$. Observe that, for every $n \geq 0$ the family of open sets $\left\{U_{\alpha}: \alpha \in B_{n}\right\}$ is pairwise disjoint by construction. Moreover, for every $n \geq$ 0 and $\alpha \in B_{n}$, by monotonicity of $\pi_{n}$ and the fact that $\bigcup\left\{\operatorname{Int} C F^{-1}\left(V_{\beta}\right): \beta \in\right.$ $\left.\pi_{n}^{-1}(\alpha)\right\}=\bigcup\left\{W_{\beta}: \beta \in \pi_{n}^{-1}(\alpha)\right\}$ is dense in $\operatorname{Int} \mathrm{Cl}^{-1}\left(V_{\alpha}\right)=W_{\alpha}$ for every $\alpha \in A_{n}$, we get $U_{\beta} \subset U_{\alpha}$ for every $\beta \in \pi_{n}^{-1}(\alpha)$. Let

$$
U_{n}:=\bigcup\left\{U_{\alpha}: \alpha \in B_{n}\right\}, \quad n \geq 0 .
$$

Each $U_{n}$ is open in $X$. We claim that $U_{n}$ are also dense in $X$.

First, observe that $\bigcup\left\{W_{\alpha}: \alpha \in A_{n}\right\}$ is dense in $X$ for every $n$. For $n=0$ this is obviously true since for $\alpha \in A_{0}, F^{-1}\left(V_{\alpha}\right)=F^{-1}(Y)=\operatorname{Dom}(F)$ and hence $W_{\alpha}=X$. For $n>0$ the density follows by induction from the assumption that $\bigcup\left\{W_{\beta}: \beta \in \pi_{n}^{-1}(\alpha)\right\}$ is dense in $W_{\alpha}$ for every $\alpha \in A_{n}$.

To prove the denseness of $U_{n}, n \geq 0$, take a nonempty open set $U \subset X$. Since $\bigcup\left\{W_{\alpha}: \alpha \in A_{n}\right\}$ is dense in $X$ there is at least one $\alpha \in A_{n}$ such that $U \cap W_{\alpha} \neq \varnothing$. Take the minimal such $\alpha$. This implies $U \cap W_{\alpha^{\prime}}=\varnothing$ for every $\alpha^{\prime}<\alpha$ and $\alpha^{\prime} \in A_{n}$. Hence $U \cap U_{\alpha} \neq \varnothing$.

Let $X_{1}:=\bigcap_{n=0}^{\infty} U_{n}$. Since $X$ is a Baire space the set $X_{1}$ is dense $G_{\delta}$ in $X$. Each $x \in X_{1}$ belongs to just one set from the disjoint family $\left\{U_{\alpha}: \alpha \in B_{n}\right\}$. Therefore, it determines in a unique way a $\pi$-chain $\left\{\alpha_{n}(x)\right\}, \alpha_{n}(x) \in B_{n}$, such that $x \in \bigcap_{n=0}^{\infty} U_{\alpha_{n}(x)}$

Consider the multivalued mapping $\Phi: X_{1} \rightarrow K(\mathbf{s})$ determined by

$$
\Phi(x):=\bigcap_{n=0}^{\infty} V_{\alpha_{n}(x)}, \quad x \in X_{1} .
$$

The mapping $\Phi$ is compact-valued and has nonempty images, i.e., $\operatorname{Dom}(\Phi)=$ $X_{1}$. Moreover, $\Phi$ is u.s.c. Indeed, let $V$ be an open subset of $Y$ such that $\Phi(x) \subset V$ for some $x \in X_{1}$. Since the sieve $\mathbf{s}$ is complete we get some $\alpha_{n}(x) \in B_{n}$ such that $V_{\alpha_{n}(x)} \subset V$. Then it is easy to check that $\Phi\left(x^{\prime}\right) \subset V_{\alpha_{n}(x)}$ for every $x^{\prime} \in U_{\alpha_{n}(x)} \cap X_{1}$. Hence $\Phi$ is u.s.c.o. in $X_{1}$.

Finally, let us define the mapping $G: X_{1} \rightarrow K(\mathbf{s})$ by

$$
G(x):=F(x) \cap \Phi(x), \quad x \in X_{1} .
$$

As an intersection of two mappings with graphs closed in $X_{1} \times Y, G$ also has a closed graph. We prove that $G$ is nonempty-valued. 
Take $x_{0} \in X_{1}$ and suppose $G\left(x_{0}\right)=\varnothing$. This means that the nonempty compact (in $X \times Y$ ) set $\left\{\left(x_{0}, y\right): y \in \Phi\left(x_{0}\right)\right\}$ does not intersect the graph of $F$. Since $\operatorname{Gr}(F)$ is a closed subset of $X \times Y$, routine considerations show that there are open subsets $U$ of $X$ and $V$ of $Y$ such that $x_{0} \in U, \Phi\left(x_{0}\right) \subset V$ and $(U \times V) \cap \operatorname{Gr}(F)=\varnothing$. That is, $F(x) \cap V=\varnothing$ for every $x \in U$. This is a contradiction since for some $n, V_{\alpha_{n}\left(x_{0}\right)} \subset V$ and, on the other hand, $U$ has a nonempty intersection with $U_{\alpha_{n}\left(x_{0}\right)} \subset W_{\alpha_{n}\left(x_{0}\right)}=\operatorname{Int} \mathrm{Cl} F^{-1}\left(V_{\alpha_{n}\left(x_{0}\right)}\right)$. Hence $G$ is nonempty-valued. This implies $X_{1} \subset \operatorname{Dom}(F)$.

Further, every nonempty-valued mapping with closed graph, which is contained in an u.s.c.o. mapping with the same domain, is u.s.c.o. itself. Hence $G$ is an u.s.c.o. selection of $F$ on $X_{1}$. The proof of the theorem is completed.

Remark 3.2. The special case when $F=f^{-1}$ for some continuous and singlevalued mapping $f: Y \rightarrow X$ and the sieve $\mathbf{s}$ is open deserves more attention. It was essentially considered by E. Michael in [M4] (see Theorem 4.12 below). It can be shown in this case, that, in addition to the conclusion of the above theorem, the set $C:=G\left(X_{1}\right)$ is a $G_{\delta}$-subset of $Y$. Indeed, with the notations in the proof above, let $H_{n}:=\bigcup\left\{f^{-1}\left(U_{\alpha}\right) \cap V_{\alpha}: \alpha \in B_{n}\right\}$. The continuity of $f$ implies that every $H_{n}$ is open in $Y$. Since for every $\pi$-chain $\left\{\alpha_{n}\right\}, \alpha_{n} \in B_{n}$, we have $F\left(\bigcap_{n=0}^{\infty} U_{\alpha_{n}}\right)=f^{-1}\left(\bigcap_{n=0}^{\infty} U_{\alpha_{n}}\right)=\bigcap_{n=0}^{\infty} f^{-1}\left(U_{\alpha_{n}}\right)$ it is easily verified that $C=\bigcap_{n=0}^{\infty} H_{n}$.

We prove further, that sometimes the selection $G$ from Theorem 3.1 coincides with the restriction of the mapping $F$ on the set $X_{1}$. That is, the mapping $F$ is itself u.s.c.o. in a dense $G_{\delta}$-subset of its domain.

Theorem 3.3. Let $F: X \rightarrow Y$ be a mapping with closed graph and $\operatorname{Dom}(F)$ be dense in $X$. Let $X$ be a Baire space and $Y$ possess a complete sieve $\mathbf{s}=\left(\left\{V_{\alpha}: \alpha \in A_{n}\right\},\left\{\pi_{n}\right\}\right)$ such that $\bigcup\left\{\operatorname{Int} F^{\#}\left(V_{\beta}\right): \beta \in \pi_{n}^{-1}(\alpha)\right\}$ is dense in Int $F^{\#}\left(V_{\alpha}\right)$ for every $\alpha \in A_{n}, n \geq 0$. Then there exists a dense $G_{\delta}$-subset $X_{1}$ of $X$ such that $X_{1} \subset \operatorname{Dom}(F)$ and the restriction $F \mid X_{1}$ of $F$ on $X_{1}$ is an u.s.c.o. mapping from $X_{1}$ into $K(\mathbf{s})$. If, in addition, for every $\pi$-chain $\left\{\alpha_{n}\right\}, \alpha_{n} \in A_{n}$, $\bigcap_{n=0}^{\infty} V_{\alpha_{n}}$ is a one-point set then at the points of $X_{1}, F$ is single-valued and u.s.c. as a mapping from $X$ into $Y$.

Proof. Let $\mathbf{s}=\left(\left\{V_{\alpha}: \alpha \in A_{n}\right\},\left\{\pi_{n}\right\}\right)$ be the complete sieve in $Y$. The proof of this theorem is similar to the proof of Theorem 3.1.

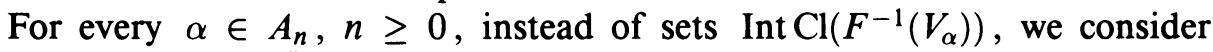
the sets $W_{\alpha}:=\operatorname{Int} F^{\#}\left(V_{\alpha}\right)$. After well-ordering the sets $A_{n}$ in a monotonic way with respect to the mappings $\pi_{n}$ we put $U_{\alpha}=W_{\alpha} \backslash \mathrm{Cl}\left(\bigcup\left\{W_{\beta}: \beta<\alpha, \beta \in A_{n}\right\}\right)$, $B_{n}:=\left\{\alpha \in A_{n}: U_{\alpha} \neq \varnothing\right\}, U_{n}:=\bigcup\left\{U_{\alpha}: \alpha \in B_{n}\right\}, n \geq 0$. In the same way as in Theorem 3.1 we prove that $U_{n}$ are dense in $X$ and hence $X_{1}:=\bigcap_{n=0}^{\infty} U_{n}$ is a dense $G_{\delta}$-subset of $X$ such that every $x \in X_{1}$ determines a unique $\pi$-chain $\left\{\alpha_{n}(x)\right\}, \alpha_{n}(x) \in B_{n}$, with $x \in \bigcap_{n=0}^{\infty} U_{\alpha_{n}(x)}$. We put $\Phi(x):=\bigcap_{n=0}^{\infty} V_{\alpha_{n}(x)}$. $\Phi$ is u.s.c.o. mapping from $X_{1}$ into $K(\mathbf{s})$. Observe that, by the construction $F\left(U_{\alpha}\right) \subset V_{\alpha}$ for every $\alpha \in B_{n}, n \geq 0$, and hence for each $x \in X_{1} F(x) \subset \Phi(x)$. Therefore $G(x):=F(x) \cap \Phi(x)=F(x)$ for every $x \in X_{1}$.

As in the proof of Theorem 3.1 one proves (using that $\operatorname{Dom}(F)$ is dense in $X$ and $\operatorname{Gr}(F)$ is closed in $X \times Y)$ that $F$ is nonempty-valued at the points of $X_{1}$. Hence $F \mid X_{1}: X_{1} \rightarrow K(\mathbf{s})$ is an u.s.c.o. mapping with nonempty values. 
Suppose, in addition, that for every $\pi$-chain $\left\{\alpha_{n}\right\} \bigcap_{n=0}^{\infty} V_{\alpha_{n}}$ is a one-point set. Then $\Phi$ is single-valued and consequently for every $x \in X_{1} F(x)=\Phi(x)$ is a singleton. Let $x_{0} \in X_{1}$ and $V$ be an open subset of $Y$ such that $F\left(x_{0}\right) \in V$. Then for some $n, V_{\alpha_{n}\left(x_{0}\right)} \subset V$. Since $F\left(U_{\alpha_{n}\left(x_{0}\right)}\right) \subset V_{\alpha_{n}\left(x_{0}\right)}$ we get that $F$ is u.s.c. at the points of $X_{1}$.

Remark 3.4. In the proof above, the closedness of $\operatorname{Gr}(F)$ and the density of $\operatorname{Dom}(F)$ in $X$ were used only to prove that the mapping $F$ is nonemptyvalued at the points of $X_{1}$. Therefore, the following modification of Theorem 3.3 is true: Let $F$ act between the Baire space $X$ and the topological space $Y$. Suppose $Y$ admits a sieve $s$ such that for every $\pi$-chain $\left\{\alpha_{n}\right\}, \alpha_{n} \in$ $A_{n}, \bigcap_{n=0}^{\infty} V_{\alpha_{n}}$ is either empty or is a one-point set such that for every open $V$ containing $\bigcap_{n=0}^{\infty} V_{\alpha_{n}}$ one has $V_{\alpha_{n}} \subset V$ for some $n$. Let $\bigcup\left\{\operatorname{Int} F^{\#}\left(V_{\beta}\right): \beta \in\right.$ $\left.\pi_{n}^{-1}(\alpha)\right\}$ be dense in Int $F^{\#}\left(V_{\alpha}\right)$ for every $\alpha \in A_{n}, n \geq 0$. Then there is a dense $G_{\delta}$-subset $X_{1}$ of $X$ such that: for every $x \in X_{1}$, either $F(x)=\varnothing$ or $F$ is single-valued and u.s.c. at $x$. In particular, if $\operatorname{Dom}(F)=X$ then $F$ is single-valued and u.s.c. at any point of $X_{1}$.

\section{SELECTION THEOREMS FOR LOWER DEMICONTINUOUS MAPPINGS}

In this section we give some important cases when Theorem 3.1 can be applied. For this we need some more notions.

There are different continuity properties of multivalued mappings which are weaker than the lower semicontinuity (see for example [Chr, ChrK, DK, K1, and $\mathrm{K} 2 \mathrm{]}$ ). In this paper we shall be concerned with a further relaxation of the lower semicontinuity. We call a mapping $F: X \rightarrow Y$ lower demicontinuous (1.d.c.) in $X$ if for every open $V$ in $Y$ the set $\left.\operatorname{Int} \mathrm{Cl}_{\left(F^{-1}\right.}(V)\right)$ is dense in $\mathrm{Cl}\left(F^{-1}(V)\right)$.

Like in the case of single-valued maps (see [HS]), we call a multivalued mapping $F: X \rightarrow Y$ demiopen if $\operatorname{Int}_{Y} \mathrm{Cl}_{Y}(F(U))$ is dense in $\mathrm{Cl}_{Y}(F(U))$, provided $U$ is open in $X$.

The next is elementary.

Proposition 4.1. The following are equivalent for $F: X \rightarrow Y$ :

(a) $F$ is lower demicontinuous in $X$;

(b) for every couple $(U, V)$ consisting of open subsets of $X$ and $Y$ respectively such that $(U \times V) \cap \operatorname{Gr}(F) \neq \varnothing$ one has $\operatorname{Int} \mathrm{Cl}\left(U \cap F^{-1}(V)\right) \neq \varnothing$;

(c) the restriction of the natural projection map $\pi_{X}: X \times Y \rightarrow X$ on $\operatorname{Gr}(F)$ is demiopen.

In other words, $F$ is lower demicontinuous in $X$ iff for every open $U$ in $X$ and open $V$ in $Y$ such that $U \cap F^{-1}(V) \neq \varnothing$, the set $F^{-1}(V)$ is dense somewhere in $U$.

Obviously, lower semicontinuity of $F$ in $X$ implies its lower demicontinuity. Moreover, it is an immediate consequence from Propositions 2.1(c) and 4.1(b) that every nonempty-valued minimal u.s.c.o. $F$ in $X$ is lower demicontinuous in $X$.

Having in mind Proposition 4.1 we get immediately its dual.

Proposition 4.2. The following are equivalent for $F: X \rightarrow Y$ :

(a) $F$ is demiopen; 
(b) $F^{-1}$ is lower demicontinuous;

(c) for every couple $(U, V)$ of open subsets of $X$ and $Y$ such that $(U \times V) \cap$ $\operatorname{Gr}(F) \neq \varnothing$ it is true that $\operatorname{Int}_{Y} \mathrm{Cl}_{Y}(V \cap F(U)) \neq \varnothing$;

(d) the restriction of the natural projection mapping $\pi_{Y}: X \times Y \rightarrow Y$ on $\operatorname{Gr}(F)$ is demiopen.

Let $Y^{\prime}$ be a nonempty subset of $Y$. We say that the multivalued mapping $F: X \rightarrow Y$ embraces $Y^{\prime}$ if for every open $W$ in $Y$ containing $Y^{\prime}$ the set $\{(x, y) \in \operatorname{Gr}(F): y \in W\}$ is dense in $\operatorname{Gr}(F)$, i.e., $\pi_{Y}^{-1}(W) \cap \operatorname{Gr}(F)$ is dense in $\operatorname{Gr}(F)$.

Proposition 4.3. The following are equivalent for $F: X \rightarrow Y$, provided $Y$ is regular:

(a) $F: X \rightarrow Y$ embraces $Y^{\prime} \subset Y$;

(b) for every open sets $V_{1}$ and $V_{2}$ in $Y$ such that $V_{1} \subset V_{2}$ and $V_{1} \cap Y^{\prime}=$ $V_{2} \cap Y^{\prime}$ the set $F^{-1}\left(V_{1}\right)$ is dense in $F^{-1}\left(V_{2}\right)$;

(c) If $\bigcup\left\{V_{\alpha}: \alpha \in A\right\} \subset V, V_{\alpha}, V$ are open in $Y$ and $V \cap Y=\bigcup\left\{V_{\alpha} \cap Y^{\prime}: \alpha \in\right.$ $A\}$ then the set $\bigcup\left\{F^{-1}\left(V_{\alpha}\right): \alpha \in A\right\}$ is dense in $F^{-1}(V)$.

Proof. (b) $\Leftrightarrow(\mathrm{c})$ is obvious. We prove $(\mathrm{a}) \Rightarrow(\mathrm{b})$. Take open subsets $V_{1}$ and $V_{2}$ of $Y$ such that $V_{1} \subset V_{2}, V_{1} \cap Y^{\prime}=V_{2} \cap Y^{\prime}$ and suppose that $F^{-1}\left(V_{2}\right) \backslash \mathrm{Cl} F^{-1}\left(V_{1}\right)$ $\neq \varnothing$. Put $H:=X \backslash \mathrm{Cl} F^{-1}\left(V_{1}\right)$ and take $x_{0} \in H \cap F^{-1}\left(V_{2}\right)$. Hence there is $y_{0} \in V_{2} \cap F\left(x_{0}\right)$. Further, there exist disjoint open sets $W_{1}$ and $W_{2}$ of $Y$ such that $y_{0} \in W_{1} \subset V_{2}$ and $Y \backslash V_{2} \subset W_{2}$. Let $W:=V_{1} \cup W_{2}$. Then, by construction $Y^{\prime} \subset W$. Consider the set $B:=\{(x, y) \in \operatorname{Gr}(F): y \in W\}$. We have $\left(H \times W_{1}\right) \cap B=\varnothing$ while $\left(x_{0}, y_{0}\right) \in\left(H \times W_{1}\right) \cap \operatorname{Gr}(F)$. This is a contradiction.

Conversely, to prove (b) $\Rightarrow(\mathrm{a})$, suppose that $W$ is an open subset of $Y$ such that $W \supset Y^{\prime}$. Let $U$ and $V$ be nonempty open subsets of $X$ and $Y$ such that $(U \times V) \cap \operatorname{Gr}(F) \neq \varnothing$. Put $V_{1}:=W \cap V$. Obviously $V_{1} \cap Y^{\prime}=V \cap Y^{\prime}$ and hence $F^{-1}\left(V_{1}\right)$ is dense in $F^{-1}(V)$. Since $U \cap F^{-1}(V) \neq \varnothing$ we get $U \cap F^{-1}\left(V_{1}\right) \neq \varnothing$. The proof is completed.

Proposition 4.4. Let the mapping $F: X \rightarrow Y$ embrace $Y^{\prime} \subset Y$ and $Y$ be regular. Then $F(X) \subset \mathrm{Cl}_{Y}\left(Y^{\prime}\right)$.

The proof is straightforward and is omitted.

The following assertion (the proof of which is elementary) gives some sufficient conditions for a mapping $F: X \rightarrow Y$ to embrace a subset $Y^{\prime}$ of $Y$.

Proposition 4.5. Each of the following conditions implies that $F$ embraces $Y^{\prime}$ :

(a) $F(X) \subset Y^{\prime}$;

(b) the set $\left\{(x, y) \in \operatorname{Gr}(F): y \in Y^{\prime}\right\}$ is dense in $\operatorname{Gr}(F)$;

(c) $Y^{\prime}$ is dense in $Y$ and the mapping $F$ is demiopen;

(d) $Y^{\prime}$ is dense in $Y$ and $F^{-1}: Y \rightarrow X$ is lower demicontinuous.

The topological space $X$ is said to be sieve complete (see [CČN and M3]) if it has a complete open sieve $\mathbf{s}=\left(\left\{V_{\alpha}: \alpha \in A_{n}\right\},\left\{\pi_{n}\right\}\right), n \geq 0$, such that $V_{\alpha}=\bigcup\left\{V_{\beta}: \beta \in \pi_{n}^{-1}(\alpha)\right\}$ for every $\alpha \in A_{n}, n \geq 0$. Every Čech complete space is sieve complete. The converse is not true in general, but the notions coincide in the class of metrizable spaces (even in paracompact spaces) [CČN, Remark 3.9d; M3, Theorem 3.2]. 
Now we are ready to prove

Theorem 4.6. Let $F$ be a lower demicontinuous mapping, acting from the Baire space $X$ into the regular space $Y$ which embraces a sieve complete subspace $Y_{1}$ of $Y$. Let $F$ be with closed graph and $\operatorname{Dom}(F)$ be dense in $X$. Then there exist a dense $G_{\delta}$-subset $X_{1}$ of $X$ and an u.s.c.o. mapping $G: X_{1} \rightarrow Y_{1}$ such that:

(a) $X_{1} \subset \operatorname{Dom}(F)$;

(b) $G$ is a selection of $F$ on the set $X_{1}$.

In particular, the requirements of this theorem are fulfilled if $F$ is 1.d.c., $\operatorname{Dom}(F)=X, \operatorname{Gr}(F)$ is closed, $X$ is Baire, and $Y$ is a Čech complete space.

Proof. Having in mind Proposition 4.4 we may assume that $Y_{1}$ is dense in $Y$. Since $Y_{1}$ is sieve complete there exists a complete open sieve $\mathbf{s}=\left(\left\{V_{\alpha}: \alpha \in\right.\right.$ $\left.\left.A_{n}\right\},\left\{\pi_{n}\right\}\right)$ in $Y_{1}$ such that $V_{\alpha}=\bigcup\left\{V_{\beta}: \beta \in \pi_{n}^{-1}(\alpha)\right\}$ for every $\alpha \in A_{n}, n \geq$ 0 . Let $\tilde{V}_{\alpha}, \alpha \in A_{n}, n \geq 0$, be open subsets of $Y$ such that $\tilde{V}_{\alpha}=Y$ for $\alpha \in A_{0}$, and for every $\alpha \in A_{n}, n \geq 0$, we have:

(1) $V_{\alpha}=Y_{1} \cap \tilde{V}_{\alpha}$ (and hence $\left.\bigcup\left\{\tilde{V}_{\beta} \cap Y_{1}: \beta \in \pi_{n}^{-1}(\alpha)\right\}=\tilde{V}_{\alpha} \cap Y_{1}\right)$;

(2) $\bigcup\left\{\tilde{V}_{\beta}: \beta \in \pi_{n}^{-1}(\alpha)\right\} \subset \tilde{V}_{\alpha}$;

(3) if $\left\{\alpha_{n}\right\}$ is a $\pi$-chain for $\mathbf{s}$ then $\bigcap_{n=0}^{\infty} \tilde{V}_{\alpha_{n}}=\bigcap_{n=0}^{\infty} V_{\alpha_{n}}$ and for every open $V$ in $Y$ such that $\bigcap_{n=0}^{\infty} \tilde{V}_{\alpha_{n}} \subset V$ one has $\tilde{V}_{\alpha_{n}} \subset V$ for some $n$.

The condition (3) is guaranteed by the regularity of $Y$ and density of $Y_{1}$ in $Y$.

The conditions (2) and (3) above show that $\tilde{\mathbf{s}}=\left(\left\{\tilde{V}_{\alpha}: \alpha \in A_{n}\right\},\left\{\pi_{n}\right\}\right)$ is a complete open sieve in $Y$. So, with a slight abuse of notation we will designate $\tilde{\mathbf{s}}$ by $\mathbf{s}$ and $\tilde{V}_{\alpha}$ by $V_{\alpha}$.

Since in our case $K(\mathbf{s}) \subset Y_{1}$, to get the conclusion of the theorem as a consequence of Theorem 3.1 we have only to show that $\bigcup\left\{\operatorname{Int} \mathrm{Cl}^{-1}\left(V_{\beta}\right): \beta \in\right.$ $\left.\pi_{n}^{-1}(\alpha)\right\}$ is dense in Int $\mathrm{Cl}^{-1}\left(V_{\alpha}\right)$ for every $\alpha \in A_{n}, n \geq 0$. But this is a straightforward consequence from (1) above, Proposition 4.3(c) (the fact that $F$ embraces $Y_{1}$ ) and lower demicontinuity of $F$.

Theorem 4.7. Let $F$ be a lower demicontinuous mapping acting from the Baire space $X$ into the regular space $Y$. Let $\operatorname{Dom}(F)$ be dense in $X, F$ be with closed graph and $F$ embrace a completely metrizable subspace $Y_{1}$ of $Y$. Then there exist a dense $G_{\delta}$-subset $X_{1}$ of $X$ and a single-valued continuous mapping $f: X_{1} \rightarrow Y_{1}$ such that $X_{1} \subset \operatorname{Dom}(F)$ and $f$ is a selection of $F$ on $X_{1}$.

Proof. Let $\rho$ be the complete metric inducing the topology in $Y_{1}$. In $Y_{1}$ we consider the following complete open sieve $\mathbf{s}$ : for $n=0$, the index set $A_{0}$ consists of only one element and $V_{\alpha}=Y_{1}, \alpha \in A_{0}$. If the sieve is constructed up to some $n, n \geq 0$, the next step is the following: for every $\alpha \in A_{n}$ consider the family $\left\{\bar{V} \subset Y_{1}: V\right.$ is open in $Y_{1}, \mathrm{Cl}_{Y_{1}}(V) \subset V_{\alpha}$ and $\rho$-diam $(V)$ $<1 /(n+1)\}$. Index that family by some set $A_{n+1}(\alpha)$ and put $A_{n+1}:=$ $\bigcup\left\{A_{n+1}(\alpha): \alpha \in A_{n}\right\}$ and $\pi_{n}^{-1}(\alpha):=A_{n+1}(\alpha)$. In this way the sieve $\mathbf{s}$ is defined and it is easily seen that $s$ is a complete open sieve in $Y_{1}$ for which, the set $\bigcap_{n=0}^{\infty} V_{\alpha_{n}}$ is a one-point set whenever $\left\{\alpha_{n}\right\}, \alpha_{n} \in A_{n}$, is a $\pi$-chain. To finish, we proceed as in the proof above. 
Call the space $X$ almost complete if it contains a dense sieve complete subspace. The class of such spaces was originally introduced in 1960 by Frolik [Fro] in a different way. If $X$ is regular, Frolik's definition is equivalent to the above one [M4, Propositions 4.2 and 4.5]. This class was studied also by Aarts and Lutzer in $[\mathrm{AL}, \S 4]$, where they called these spaces almost Čech complete. It is true that (see [Fro]) a completely regular space $X$ is almost complete iff it contains a dense and Cech complete subspace. For a detailed study of almost complete spaces (as well as spaces admitting similar completeness properties) we refer to the recent paper of E. Michael [M4]. Let us mention that every regular almost complete space is a Baire space (see, e.g., [M4, Proposition 4.5]).

We have the following important partial cases of the above theorems.

Theorem 4.8. Let $F$ be a l.d.c. and demiopen mapping from the Baire space $X$ into the regular almost complete space $Y$. Let $F$ be with closed graph and $\operatorname{Dom}(F)$ be dense in $X$. Then there exist a dense $G_{\delta}$-subset $X_{1}$ of $X$ and an u.s.c.o. mapping $G: X_{1} \rightarrow Y$ such that:

(a) $X_{1} \subset \operatorname{Dom}(F)$;

(b) $G$ is a selection of $F$ on $X_{1}$.

Proof. Since $F$ is demiopen it embraces every dense subspace of $Y$ (Proposition 4.5(c)). Therefore, we can apply Theorem 4.6.

Analogously, by Theorem 4.7 and Proposition 4.5(c) one obtains

Theorem 4.9. In the assumptions of Theorem 4.8 suppose that $Y$ contains a dense and completely metrizable subspace. Then the selection $G$ can be considered to be single-valued.

We give now some examples showing that the assumptions on $F$ in Theorem 4.6 and Theorem 4.7 are essential.

Example 4.10. Let the segment $X:=[0,1]$ be endowed with the usual topology on the real line. Let further $B \subset[0,1]$ be such that both $B$ and $X \backslash B$ are dense Baire subspaces of $X$ (e.g., let $B$ be a Bernstein subset of $[0,1]$, see [En, 4.5.5(b) and 5.5.4]). Put $Y:=(B \times\{0\}) \cup((X \backslash B) \times\{1\}) \cup(X \times(0,1))$, where $(0,1)$ is the open interval, and consider on $Y$ the topology inherited by the product topology of $X \times X$. Let $F: X \rightarrow Y$ be defined by $F(x)=(x, 0)$ if $x \in B$ and $F(x)=(x, 1)$ if $x \in X \backslash B . F$ has a closed graph because $B \times\{0\}$ and $(X \backslash B) \times\{1\}$ are closed subsets of $Y . F$ is obviously lower demicontinuous. But there is no dense $G_{\delta}$-subset of $X$ on which $F$ possesses a continuous selection. In this example $F$ does not embrace any completely metrizable subspace of $Y$.

Example 4.11. Let $X$ and $B$ be as in Example 4.10. Define $F: X \rightarrow\{0,1\}$ by $F(x)=1$ if $x \in B$ and $F(x)=0$ otherwise. $F$ is 1.d.c. but $\operatorname{Gr}(F)$ is not closed and $F$ does not have a continuous selection on a dense $G_{\delta}$-subset of $X$.

We give some further consequences of Theorem 4.6 and Theorem 4.7. Let us start with a result of Michael [M4].

Theorem 4.12 [M4, Theorem 7.2]. Let $f: Y \rightarrow X$ be a continuous and demiopen single-valued mapping acting from the regular almost complete space $Y$ 
into the space $X$ such that $f(Y)$ is dense in $X$. Then there exist a $G_{\delta}$-subset $C$ of $Y$ and a dense $G_{\delta}$-subset $D$ of $X$ such that $f \mid C: C \rightarrow D$ is perfect and onto.

Proof. Let $F:=f^{-1}: X \rightarrow Y$. By the assumptions $\operatorname{Dom}(F)=f(Y)$ is dense in $X$ and by [M4, Proposition 6.6], $X$ is a Baire space. Since $f$ is demiopen then $F$ is l.d.c. On the other hand, the continuity of $f$ implies that $F$ is open and has closed graph. Therefore, by Theorem 4.8 there are a dense $G_{\delta}$-subset $D$ of $X$ and an u.s.c.o. $G: D \rightarrow Y$ such that $D \subset \operatorname{Dom}(F)=f(Y)$ and $G$ is a selection of $F$. Let $C:=G(D)$. Since $F=f^{-1}$ and $f$ is single-valued then $F\left(x_{1}\right) \cap F\left(x_{2}\right)=\varnothing$ for $x_{1} \neq x_{2}$. Hence $G(x)=F(x) \cap C$ for every $x \in D$. This implies that $G=(f \mid C)^{-1}$ showing that $f \mid C: C \rightarrow D$ is perfect and onto. The rest follows by Remark 3.2.

Theorem 4.13 [M4, Theorem 7.3]. If the space $Y$ in Theorem 4.12 contains a dense and completely metrizable subspace then $f \mid C$ can be considered to be a homeomorphism.

Proof. As above using Theorem 4.9.

Remark 4.14. It should be noted that Theorems 7.2 and 7.3 from the paper of E. Michael [M4] contain additional information about the sets $C$ and $D$ (special completeness properties). These completeness properties do not follow directly from our Theorems 4.8 and 4.9.

In connection with Theorem 4.13 the following questions were raised in [M4, Question 7.4]: Let $f$ be an open and continuous single-valued mapping from a Čech complete space $Y$ onto a regular (or even metrizable) space $X$. Must $f$ map some nonempty subset $C$ of $Y$ homeomorphically onto a dense $G_{\delta}$ subset of $X$ ? We give here a negative answer to one of these questions, namely, to the case when $X$ is not metrizable.

Example 4.15. Let $\tau$ be a cardinal which is greater than or equal to the first uncountable cardinal. By a result of Pasinkov [Pas, Theorem 2], there are a compact space $Y_{\tau}$ of weight $\tau$ with $\operatorname{dim} Y_{\tau}=1$, and a continuous and open mapping $f$ which maps $Y_{\tau}$ onto $[0,1]^{\tau}$ and such that $\operatorname{dim}\left(f^{-1}(x)\right)=0$ for every $x \in[0,1]^{\tau}$. Let us observe that if $H$ is a nonempty $G_{\delta}$-subset of $[0,1]^{\tau}$ then $H$ contains a homeomorphic image of $[0,1]^{\tau}$. Suppose now that there is $C \subset Y_{\tau}, C \neq \varnothing$, such that $f(C)=H, H$ is $G_{\delta}$ in $[0,1]^{\tau}$, and $f \mid C$ is a homeomorphism. Then $C$ contains a homeomorphic image of $[0,1]^{\tau}$, so the same does $Y_{\tau}$. But $\operatorname{dim}[0,1]^{\tau}=\infty$ while $\operatorname{dim} Y_{\tau}=1$. This is a contradiction.

At the end of this section we show that when the range space $Y$ is second countable the conclusion of Theorem 4.7 can be obtained under weakened assumptions on the mapping $F$.

Theorem 4.16. Let $F$ be a mapping with closed graph acting from a Baire space $X$ into the completely metrizable separable space $Y$. Let $\operatorname{Dom}(F)=X$. Then there exist a dense $G_{\delta}$-subset $X_{1}$ of $X$ and a single-valued continuous mapping $f: X_{1} \rightarrow Y$ which is a selection of $F$ on $X_{1}$.

Proof. We follow a scheme from [K2]. Let $\left\{V_{n}\right\}, n \geq 1$, be a countable base for the topology in $Y$. Consider the sets $H_{n}=\mathrm{Cl} F^{-1}\left(V_{n}\right) \backslash \operatorname{Int~Cl~} F^{-1}\left(V_{n}\right)$. The sets $H_{n}$ are closed and nowhere dense in $X$ and consequently the set 
$X^{\prime}:=\bigcap_{n=1}^{\infty} X \backslash H_{n}$ is dense $G_{\delta}$ in $X$. Further, since $\left\{V_{n}\right\}, n \geq 1$, is a base in $Y$, it is routine matter to check that the restriction $F \mid X^{\prime}$ of the mapping $F$ on $X^{\prime}$ is l.d.c. To complete the proof, apply Theorem 4.7 for the mapping $F \mid X^{\prime}: X^{\prime} \rightarrow Y$.

\section{GENERIC CONTINUITY OF MULTIVALUED MAPPINGS}

In this section we prove several results in which a mapping $F: X \rightarrow Y$ is u.s.c.o. (or single-valued and u.s.c.) itself at the points of a residual subset of its domain. We start with a general result when the range space is metric.

A mapping $F: X \rightarrow Y$, where $Y$ is a metric space with a metric $\rho$, is said to be fragmented by $\rho$ (see [HJT, p. 217]) if for every $\varepsilon>0$ and every nonempty open set $U$ in $X$ there exists a nonempty open $U^{\prime} \subset U$ such that $\rho$-diam $\left(F\left(U^{\prime}\right)\right)<\varepsilon$.

Theorem 5.1. Let $F: X \rightarrow Y$, where $X$ is a Baire space and $(Y, \rho)$ is a metric space, be fragmented by the metric $\rho$. Then there exists a dense $G_{\delta}$-subset $X_{1}$ of $X$ such that:

(a) for every $x \in X_{1}$ either $F(x)=\varnothing$ or $F$ is single-valued and u.s.c. at $x$.

(b) if $\rho$ is complete, $\operatorname{Dom}(F)$ is dense in $X$ and $F$ has a closed graph then $X_{1} \subset \operatorname{Dom}(F)$ and at the points of $X_{1} F$ is single-valued and u.s.c.o.

Proof. We construct a sieve $\mathbf{S}$ (not necessarily open) in $Y$ as in the proof of Theorem 4.7. The only difference is the following: on the $(n+1)$ th step for every $\alpha \in A_{n}$ we consider the family $\left\{V: \mathrm{Cl}_{Y}(V) \subset V_{\alpha}\right.$ and $\rho$-diam $(V)<$ $1 /(n+1)\}$, i.e., here we do not require that the sets $V$ are open in $Y$. Observe that if the metric $\rho$ is complete then the sieve $\mathbf{s}$ is complete too. For every $\pi$-chain $\left\{\alpha_{n}\right\}, \alpha_{n} \in A_{n}, \bigcap_{n=0}^{\infty} V_{\alpha_{n}}$ is either empty or is a one-point set. In the latter case for every open $V$ in $Y$ containing $\bigcap_{n=0}^{\infty} V_{\alpha_{n}}$ one has $V_{\alpha_{n}} \subset V$ for some $n$.

The so constructed sieve $\mathbf{s}$, the mapping $F$ and the spaces $X$ and $Y$ satisfy the requirements of Theorem 3.3 (see also Remark 3.4). The proof is completed.

Theorem 5.2. Let $F$ be a multivalued mapping acting from a Baire space $X$ into the regular space $Y$ such that $\operatorname{Dom}(F)$ is dense in $X, \operatorname{Gr}(F)$ is closed and $F$ embraces some sieve complete subspace $Y_{1}$ of $Y$. Suppose that Int $F^{*}(V)$ is dense in $F^{-1}(V)$ for every open $V$ in $Y$. Then there exists a dense $G_{\delta}$-subset $X_{1}$ of $X$ such that $X_{1} \subset \operatorname{Dom}(F), F$ maps $X_{1}$ into $Y_{1}$, and at the points of $X_{1} F$ is u.s.c.o.

Proof. As in the proof of Theorem 4.6 we may suppose that $Y_{1}$ is dense in $Y$ and get a complete open sieve $\mathbf{s}=\left(\left\{V_{\alpha}: \alpha \in A_{n}\right\},\left\{\pi_{n}\right\}\right)$ in $Y$ such that for every $\alpha \in A_{n}, n \geq 0, \bigcup\left\{V_{\beta} \cap Y_{1}: \beta \in \pi_{n}^{-1}(\alpha)\right\}=V_{\alpha} \cap Y_{1}$ and $K(\mathbf{s}) \subset Y_{1}$. Since $F$ embraces $Y_{1}$ we have, by Proposition 4.3(c), that for every $\alpha \in A_{n}, n \geq$ $0, \bigcup\left\{F^{-1}\left(V_{\beta}\right): \beta \in \pi_{n}^{-1}(\alpha)\right\}$ is dense in $F^{-1}\left(V_{\alpha}\right)$. Now, since $\operatorname{Dom}(F)$ is dense in $X$ and Int $F^{*}(V)$ is dense in $F^{-1}(V)$ for every open $V$ in $Y$, it is easy to see that $\bigcup\left\{\operatorname{Int} F^{\#}\left(V_{\beta}\right): \beta \in \pi_{n}^{-1}(\alpha)\right\}$ is dense in Int $F^{\#}\left(V_{\alpha}\right)$. Hence, by Theorem 3.3 there is a dense $G_{\delta}$-subset $X_{1}$ of $X$ such that $X_{1} \subset \operatorname{Dom}(F)$ and $F \mid X_{1}: X_{1} \rightarrow Y_{1}$ is u.s.c.o.

We prove now that at the points of $X_{1}$ the mapping $F: X \rightarrow Y$ is u.s.c. Take $x_{0} \in X_{1}$ and let $V$ be an open subset of $Y$ such that $F\left(x_{0}\right) \subset V$. Since $F\left(x_{0}\right)$ 
is compact and $Y$ is regular there is an open subset $W$ of $Y$ with $F\left(x_{0}\right) \subset W$ and $\mathrm{Cl}_{Y}(W) \subset V$. Having in mind that $F$ is u.s.c. in $X_{1}$ there is an open subset $U$ of $X$ such that $F(x) \subset W$ for every $x \in U \cap X_{1}$. Suppose there is a point $x_{1} \in U \cap \operatorname{Dom}(F)$ such that $F\left(x_{1}\right) \backslash \mathrm{Cl}_{Y}(W) \neq \varnothing$. Take $y_{0}$ from this set. Then there is an open in $Y$ set $V_{0}$ such that $y_{0} \in V_{0}$ and $V_{0} \cap \mathrm{Cl}_{Y}(W)=\varnothing$. Since Int $F^{\#}\left(V_{0}\right)$ is dense in $F^{-1}\left(V_{0}\right)$ there exists a nonempty open $U^{\prime} \subset U$ such that $F\left(U^{\prime}\right) \subset V_{0}$. This is a contradiction since $U^{\prime} \cap X_{1}$ is a nonempty subset of $U \cap X_{1}$. The proof is completed.

In the same way one can prove

Theorem 5.3. Under the assumptions of Theorem 5.2 suppose in addition that $Y_{1}$ is completely metrizable. Then $F$ maps $X_{1}$ into $Y_{1}$ and $F$ is single-valued and u.s.c. at the points of $X_{1}$.

Let us mention that Remark 3.4 is valid also for Theorem 5.3.

We prove now another result of the above type. The proof is again based on the construction of inscribed families of disjoint open sets and it is a generalization of a construction from [KG].

Theorem 5.4. Let $X$ and $Y$ be regular and contain completely metrizable dense subspaces $X_{0} \subset X$ and $Y_{0} \subset Y$. Let $F: X \rightarrow Y$ be a nonempty-valued mapping such that for every nonempty open $V$ in $Y$ and $U$ in $X$ the sets $\operatorname{Int}_{X} F^{\#}(V)$ and $\operatorname{Int}_{Y}\left(F^{-1}\right)^{\#}(U)$ are nonempty. Then there are dense $G_{\delta}$-subsets $X_{1}$ of $X$ and $Y_{1}$ of $Y$ such that:

(a) $X_{1} \subset X_{0}$ and $Y_{1} \subset Y_{0}$;

(b) $F$ is single-valued and u.s.c. at the points of $X_{1}$ and $F^{-1}$ is single-valued and u.s.c. at the points of $Y_{1}$;

(c) the restriction $F \mid X_{1}$ is a homeomorphism between $X_{1}$ and $Y_{1}$.

Proof. It is clear that $\operatorname{Dom}\left(F^{-1}\right)=F(X)$ is dense in $Y$.

Let $X_{0} \subset X$ and $Y_{0} \subset Y$ be dense subsets of $X$ and $Y$ which are completely metrizable by some metrics $\rho$ and $d$ respectively. Take a maximal disjoint family $\Delta_{1}:=\left\{V: V\right.$ is open in $Y, d$-diam $\left.\left(V \cap Y_{0}\right)<1\right\}$. Hence $V_{1}:=\bigcup\left\{V: V \in \Delta_{1}\right\}$ is dense in $Y$. In $X$ consider the disjoint family $\Gamma_{1}^{\prime}:=\left\{\operatorname{Int}_{X} F^{\#}(V): V \in \Delta_{1}\right\}$. We prove that $\bigcup\left\{U: U \in \Gamma_{1}^{\prime}\right\}$ is dense in $X$. Let $W$ be a nonempty open subset of $X$. Then $\operatorname{Int}_{Y}\left(F^{-1}\right)^{\#}(W) \neq \varnothing$. Hence $H:=V \cap \operatorname{Int}_{Y}\left(F^{-1}\right)^{\#}(W)$ is a nonempty open subset of $Y$ for some $V \in \Delta_{1}$. This implies $\varnothing \neq \operatorname{Int}_{X} F^{\#}(H) \subset \operatorname{Int}_{X} F^{\#}(V)$. Since $F$ is nonempty-valued we have $\varnothing \neq \operatorname{Int}_{X} F^{\#}(H) \subset F^{-1}(H) \subset W$ and consequently $W \cap \operatorname{Int}_{X} F^{\#}(V) \neq \varnothing$.

Let us inscribe in $\Gamma_{1}^{\prime}$ a maximal disjoint family $\Gamma_{1}:=\{U \subset X: U$ is open in $X, \mathrm{Cl}_{X}(U) \subset U^{\prime}$ for some $U^{\prime} \in \Gamma_{1}^{\prime}$ and $\rho$-diam $\left.\left(U \cap X_{0}\right)<1\right\}$. Since $\bigcup\left\{U^{\prime}: U^{\prime} \in \Gamma_{1}^{\prime}\right\}$ is dense in $X$ the same is true for $U_{1}:=\bigcup\left\{U: U \in \Gamma_{1}\right\}$.

Now, consider $\Delta_{1}^{\prime}:=\left\{\operatorname{Int}_{Y}\left(F^{-1}\right)^{\#}(U): U \in \Gamma_{1}\right\}$. This family is again disjoint and since the union of the elements of $\Gamma_{1}$ is dense in $X$ one can prove, as above (using the fact that $\operatorname{Dom}\left(F^{-1}\right)$ is dense in $Y$ ), that $\bigcup\left\{H: H \in \Delta_{1}^{\prime}\right\}$ is dense in $Y$. Obviously for every $H \in \Delta_{1}^{\prime}$, there exists some $V \in \Delta_{1}$ such that $H \cap V \neq \varnothing$. Since $Y$ is regular, this allows to inscribe simultaneously in $\Delta_{1}^{\prime}$ and $\Delta_{1}$ a maximal disjoint family $\Delta_{2}:=\{V: V$ is open in $Y, d-\operatorname{diam}\left(V \cap Y_{0}\right)<1 / 2, V \subset H$ for some $H \in \Delta_{1}^{\prime}$ and $\mathrm{Cl}_{Y}(V) \subset V^{\prime}$ for some $\left.V^{\prime} \in \Delta_{1}\right\}$. By the maximality of $\Delta_{2}$ and the fact that $\bigcup\left\{V: V \in \Delta_{1}\right\}$ 
and $\bigcup\left\{V: V \in \Delta_{1}^{\prime}\right\}$ are dense in $Y$, it follows that $V_{2}:=\bigcup\left\{V: V \in \Delta_{2}\right\}$ is dense in $Y$. Proceeding in this way we obtain sequences of disjoint families $\left\{\Gamma_{n}\right\}$ and $\left\{\Delta_{n}\right\}$ of open sets in $X$ and $Y$ respectively such that:

(i) $U_{n}:=\bigcup\left\{U: U \in \Gamma_{n}\right\}$ and $V_{n}:=\bigcup\left\{V: V \in \Delta_{n}\right\}$ are dense in $X$ and $Y$ respectively for every $n$;

(ii) for every $U \in \Gamma_{n+1}$ and $V \in \Delta_{n+1}$ there are $U^{\prime} \in \Gamma_{n}$ and $V^{\prime} \in \Delta_{n}$ such that $\mathrm{Cl}_{X}(U) \subset U^{\prime}$ and $\mathrm{Cl}_{Y}(V) \subset V^{\prime}$;

(iii) for every $U \in \Gamma_{n}$ there is $V \in \Delta_{n}$ such that $F(U) \subset V$ and for every $V \in \Delta_{n+1}$ there is $U \in \Gamma_{n}$ such that $F^{-1}(V) \subset U$;

(iv) for every $U \in \Gamma_{n}, V \in \Delta_{n} \rho-\operatorname{diam}\left(U \cap X_{0}\right)<1 / n$ and $d$-diam $\left(V \cap Y_{0}\right)$ $<1 / n$.

Let $X_{1}:=\bigcap_{n=1}^{\infty} U_{n}$ and $Y_{1}:=\bigcap_{n=1}^{\infty} V_{n}$. Obviously $X_{1}$ and $Y_{1}$ are dense $G_{\delta}$-subsets of $X$ and $Y$. Moreover, (iv) and the regularity of $X$ and $Y$ imply that $X_{1} \subset X_{0}$ and $Y_{1} \subset Y_{0}$.

Each $x \in X_{1}$ defines in a unique way sequences $\left\{U_{n}(x)\right\}$ and $\left\{V_{n}(x)\right\}$ such that $U_{n}(x) \in \Gamma_{n}, V_{n}(x) \in \Delta_{n}, x \in \bigcap_{n=1}^{\infty} U_{n}(x), \mathrm{Cl}_{X}\left(U_{n+1}(x)\right) \subset U_{n}(x)$, $\mathrm{Cl}_{Y}\left(V_{n+1}(x)\right) \subset V_{n}(x), F\left(U_{n}(x)\right) \subset V_{n}(x)$, and $F^{-1}\left(V_{n+1}(x)\right) \subset U_{n}(x)$. Moreover, since $\rho$-diam $\left(U_{n}(x) \bigcap X_{0}\right)<1 / n$ and $d$-diam $\left(V_{n}(x) \cap Y_{0}\right)<1 / n$ the sets $\bigcap_{n=1}^{\infty} U_{n}(x)$ and $\bigcap_{n=1}^{\infty} V_{n}(x)$ are one-point subsets of $X_{1}$ and $Y_{1}$ respectively. Hence $F(x) \subset \bigcap_{n=1}^{\infty} V_{n}(x)$ and since the latter set is a singleton and $F$ is nonempty-valued, it follows that $F$ is single-valued at the points of $X_{1}$.

Take further some $x_{0} \in X_{1}$. Then $F\left(x_{0}\right)=\bigcap_{n=1}^{\infty} V_{n}\left(x_{0}\right)$. Let $F\left(x_{0}\right) \in V$ for some open $V$ in $Y$. The sequence $\left\{V_{n}\left(x_{0}\right) \cap Y_{0}\right\}$ is a local base for the point $F\left(x_{0}\right)$ in $Y_{0}$. Since $Y_{0}$ is dense in $Y$ and $Y$ is regular it follows that $\left\{V_{n}\left(x_{0}\right)\right\}$ is a base for $F\left(x_{0}\right)$ in $Y$. Therefore $V_{n}\left(x_{0}\right) \subset V$ for some $n$. Now, for every $x \in U_{n}\left(x_{0}\right)$ we have $F(x) \subset V_{n}\left(x_{0}\right)$. Hence $F$ is u.s.c. at $x_{0}$.

Finally, we prove that $F\left(X_{1}\right)=Y_{1}$ and $F^{-1}$ is single-valued and u.s.c. at the points of $Y_{1}$. Take $y \in Y_{1}$. This $y$ determines (in a unique way) sequences $\left\{U_{n}(y)\right\},\left\{V_{n}(y)\right\}$ with the same properties as above. Now, by the construction $\bigcap_{n=1}^{\infty} U_{n}(y)$ is a one-point set in $X_{0}$, say $x$, and as seen from above $F(x)=\{y\}$. Hence $F \mid X_{1}: X_{1} \rightarrow Y_{1}$ is onto and, again as above, $F^{-1}$ is single-valued and u.s.c. at the points of $Y_{1}$. As a result, the restriction $F \mid X_{1}$ is a homeomorphism between $X_{1}$ and $Y_{1}$.

Remark 5.5. It can be seen that Theorem 5.4 is true if $\operatorname{Dom}(F)$ is dense in $X$ (rather than $\operatorname{Dom}(F)=X$ ) provided $\operatorname{Gr}(F)$ is closed in $X \times Y$ (see Theorem 3.3 and Remark 3.4).

\section{SOME OTHER APPLICATIONS.}

This section illustrates how the results obtained in the previous sections can be used in order to prove some known as well as new results. We start with two results concerning generic well-posedness of optimization problems.

(a) Generic well-posedness of optimization problems. Let $X$ be a completely regular space and $C(X)$ denotes the space of all continuous real-valued and bounded functions on $X$ endowed with the usual sup-norm $\|f\|=\sup \{|f(x)|$ : $x \in X\}$. The couple $(X, f)$ can be considered as a maximization problem: 
find $x_{0} \in X$ such that $f\left(x_{0}\right)=\sup \{f(x): x \in X\}=: \sup (X, f)$. The maximization problem $(X, f)$ is said to be Tikhonov well-posed [T] if it has unique solution and every maximizing sequence $\left\{x_{n}\right\}$ (that is $f\left(x_{n}\right) \rightarrow \sup (X, f)$ ) converges to this solution. $(X, f)$ is generalized Tikhonov well-posed (see [FV] for the case of a metric space $X$ and [ČKR1, ČKR2] for the general case of a topological space) if every maximizing net has a convergent subnet. Let $e(f), f \in C(X)$, denote the unique continuous extension of $f$ on $\beta X$, the Stone-Cech compactification of $X$. Let further, $M: C(X) \rightarrow \beta X$ be the multivalued mapping defined by $M(f):=\{x \in \beta X: e(f)(x)=\sup (X, f)\}, f \in$ $C(X)$. It is well-known (see e.g. [ČK $1, \check{C} K 2]$ ) that $M$ is open minimal and u.s.c.o. It is proved in [ČKR1, ČKR2, Propositions 1.10 and 1.12], that $(X, f)$ is Tikhonov well-posed (resp. generalized Tikhonov well-posed) iff the set $M(f)$ is a singleton, necessarily lying in $X$ (resp. $M(f) \subset X$ ).

Put $T:=\{f \in C(X):(X, f)$ is Tikhonov well-posed $\}$ and $G T:=\{f \in$ $C(X):(X, f)$ is generalized Tikhonov well-posed $\}$. For compact $X$ the following theorem was proved in [ČK1, Proposition 1 and Theorem 5], [ČK2, Proposition 1.2 and Theorem 2.3].

Theorem 6.1 [ČKR1, ČKR2, Theorem 3.5]. The set $T$ contains a dense $G_{\delta^{-}}$ subset of $C(X)$ iff $X$ contains a dense and completely metrizable subspace.

Proof. Suppose that $T$ contains a dense $G_{\delta}$-subset $T_{1}$ of $C(X)$. Consider the mapping $M^{-1}: \beta X \rightarrow C(X)$. It is l.s.c. (since $M$ is open) and has closed graph (since $M$ is u.s.c.o.). Moreover, by Proposition 4.5(d), $M^{-1}$ embraces every dense subset of $C(X)$, in particular $T_{1}$. Hence, by Theorem 4.7 there exist a dense $G_{\delta}$-subset $X_{1}$ of $\beta X$ and a continuous single-valued mapping $h: X_{1} \rightarrow T_{1}$ which is a selection of $M^{-1}$ on $X_{1}$. Obviously $X_{1}$ is Čech complete. Take $x \in X_{1}$. Then $h(x) \in T_{1}$ and hence $h(x) \in T$. Therefore $M(h(x)) \subset X$, showing that $x \in X$. Hence $X_{1} \subset X$. On the other hand, since $h$ is a selection of $M^{-1}$ mapping $X_{1}$ into $T_{1}$ and $M$ is u.s.c.o. and single-valued on $T$, it is seen that $h$ is a homeomorphism between $X_{1}$ and $h\left(X_{1}\right) \subset T_{1}$. To finish, let us recall that every metrizable Čech complete space is completely metrizable.

Conversely, let $X$ contain a dense and completely metrizable subspace $X_{1}$. Apply Theorem 5.3 to the mapping $M: C(X) \rightarrow \beta X$. Hence there is a dense $G_{\delta}$-subset $T_{1}$ of $C(X)$ on which $M$ is single-valued. Hence $T_{1} \subset T$.

Theorem 6.2 [ČKR1, ČKR2, Theorem 2.3]. The set $G T$ contains a dense $G_{\delta^{-}}$ subset of $C(X)$ iff $X$ is almost complete.

Proof. Let $G T$ contain a dense $G_{\delta}$-subset $T_{1}$ of $C(X)$. As in the previous proof apply Theorem 4.6 to the mapping $M^{-1}: \beta X \rightarrow C(X)$. We get a dense $G_{\delta}$-subset $X_{1}$ of $\beta X$ (hence $X_{1}$ is Cech complete) such that $X_{1} \subset X$.

Conversely, let $X$ be almost complete. Then (see $\S 4) X$ contains a dense and Čech complete subspace $X_{1}$. By Theorem 5.2 (applied to the mapping $M: C(X) \rightarrow \beta X)$ there is a dense $G_{\delta}$-subset $T_{1}$ of $C(X)$ such that $M$ maps $T_{1}$ into $X_{1}$. Since $X_{1} \subset X$ we get $T_{1} \subset G T$. The proof is completed.

Remark 6.3. Since for any $x \in \beta X, M^{-1}(x)$ is a convex closed subset of $C(X)$ and $M^{-1}$ is 1.s.c. in $\beta X$, the classical Michael selection theorem [M1] always 
gives a single-valued selection of $M^{-1}: \beta X \rightarrow C(X)$ which is defined on $\beta X$. The values of this selection, however, are not obliged to lie in $T$ or $G T$.

(b) A generalized Lavrentieff theorem. The classical Lavrentieff theorem asserts that a homeomorphism $f$ between $A$ and $B$, where $A$ and $B$ are subsets of the complete metric spaces $X$ and $Y$ correspondingly, can be extended to a homeomorphism $f^{\prime}$ between some $G_{\delta}$-subsets $A^{\prime} \subset \mathrm{Cl}_{X}(A)$ and $B^{\prime} \subset \mathrm{Cl}_{Y}(B)$, with $A \subset A^{\prime}$ and $B \subset B^{\prime}$. In [BLL] a class of spaces is characterized for which the Lavrentieff theorem is valid. This happens if every closed subset $C$ of $X$ (and $D$ of $Y$ ) is Čech complete and has a $G_{\delta}$-diagonal in $C \times C($ in $D \times D)$. We slightly change this setting of the problem and look for an extension $f^{\prime \prime}$ which is a homeomorphism between certain $G_{\delta}$-subsets $A^{\prime \prime}$ of $\mathrm{Cl}_{X}(A)$ and $B^{\prime \prime}$ of $\mathrm{Cl}_{Y}(B)$ but these latter sets do not necessarilly contain $A$ and $B$. Precisely, $f^{\prime \prime}$ is a homeomorphism between residual subsets of $\mathrm{Cl}_{X}(A)$ and $\mathrm{Cl}_{Y}(B)$ which contain $A$ and $B$. In this case the corresponding Lavrentieff type theorem is true for a class of spaces which is strictly larger than that described in [BLL].

A completely regular topological space $X$ is said to be from the class $\mathbf{M}$ if every closed subset of $X$ contains a dense and completely metrizable subspace.

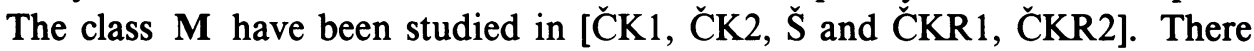
exist Eberlein compacts (that is compact spaces which are homeomorphic to weak compact sets in a Banach space) which do not have a $G_{\delta}$-diagonal. For example, take the one-point Aleksandroff compactification of the interval $[0,1]$ where all points are open sets. By the criterion of Rosenthal [Ro] this space is an Eberlein compact, but its diagonal is not $G_{\delta}$. On the other hand, Eberlein compacta are in the class $\mathbf{M}$ (see $[\mathrm{Na}]$ ).

We now can state the following Lavrentieff type theorem:

Theorem 6.4. Let $X$ and $Y$ be from the class $M$ and $f$ be a homeomorphism between $A \subset X$ and $B \subset Y$. Then $f$ can be extended to a homeomorphism $f^{\prime}$ between residual subsets of $\mathrm{Cl}_{X}(A)$ and $\mathrm{Cl}_{Y}(B)$ containing $A$ and $B$. Moreover, dense and completely metrizable subsets $A^{\prime} \subset \mathrm{Cl}_{X}(A)$ and $B^{\prime} \subset \mathrm{Cl}_{Y}(B)$ exist (not necessarily containing $A$ and $B$ ) which are homeomorphic under $f^{\prime}$.

Proof. Take $\mathrm{Cl}_{X}(A)$ and $\mathrm{Cl}_{Y}(B)$ in $X$ and $Y$ respectively. Then $\mathrm{Cl}_{X}(A)$ and $\mathrm{Cl}_{Y}(B)$ contain dense and complete metrizable subspaces $A_{0}$ and $B_{0}$ respectively.

Define $F: \beta \mathrm{Cl}_{X}(A) \rightarrow \beta \mathrm{Cl}_{Y}(B)$ by putting $\mathrm{Gr}(F)=\mathrm{Cl}(\mathrm{Gr}(f)) . \quad F$ and $F^{-1}$ are nonempty-valued since $\operatorname{Dom}(f)=A$ and $\operatorname{Dom}\left(f^{-1}\right)=B$ are dense in the compact spaces $\beta \mathrm{Cl}_{X}(A)$ and $\beta \mathrm{Cl}_{Y}(B)$. Moreover, both $F$ and $F^{-1}$ are u.s.c.o. Further, since $f$ is a homeomorphism between $A$ an $B$ it is easily checked that $\operatorname{Int} F^{\#}(V)$ and $\operatorname{Int}\left(F^{-1}\right)^{\#}(U)$ are nonempty in $\beta \mathrm{Cl}_{X}(A)$ and $\beta \mathrm{Cl}_{Y}(B)$ provided $U$ and $V$ are nonempty open subsets in $\beta \mathrm{Cl}_{X}(A)$ and $\beta \mathrm{Cl}_{Y}(B)$ respectively. Hence, Theorem 5.4 gives the existence of dense $G_{\delta}$-subsets $A^{\prime}$ of $\beta \mathrm{Cl}_{X}(A)$ and $B^{\prime}$ of $\beta \mathrm{Cl}_{Y}(B)$ such that $F \mid A^{\prime}$ is a homeomorphism between $A^{\prime}$ and $B^{\prime}$. Observe that (again by Theorem 5.4) $A^{\prime} \subset A_{0}$ and $B^{\prime} \subset B_{0}$, hence $A^{\prime}$ and $B^{\prime}$ are also completely metrizable.

Since $f$ is a homeomorphism the restrictions of $F$ on $A$ and of $F^{-1}$ on $B$ coincide with $f$ and $f^{-1}$ respectively. Put $A^{\prime \prime}:=A \cup A^{\prime}$ and $B^{\prime \prime}:=B \cup B^{\prime}$. $A^{\prime \prime}$ and $B^{\prime \prime}$ are residual subsets of $\mathrm{Cl}_{X}(A)$ and $\mathrm{Cl}_{Y}(B)$ containing $A$ and 
$B$. To finish, remember that $F$ and $F^{-1}$ are u.s.c.o. Therefore $F \mid A^{\prime \prime}$ is a homeomorphism between $A^{\prime \prime}$ and $B^{\prime \prime}$.

(c) Generic differentiability of convex functions. Let $(E,\|\cdot\|)$ designate a real Banach space with norm $\|\cdot\|$. By $E^{*}$, as usual, we denote the dual of $E$. That is, $E^{*}$ is the space of all continuous linear functionals in $E$ endowed with the dual norm $\left\|x^{*}\right\|=\sup \left\{\left|\left\langle x, x^{*}\right\rangle\right|: x \in B\right\}, x^{*} \in E^{*}$, where $B:=\{x \in$ $E:\|x\| \leq 1\}$ is the closed unit ball in $E$ and $\langle\cdot, \cdot\rangle$ designates the usual duality between $E$ and $E^{*}$. The weak star topology in $E^{*}$ will be denoted by $w^{*}$.

Let $f: E \rightarrow \mathbf{R}$ be a continuous convex function. The subdifferential $\partial_{f}$ of $f$, is a multivalued mapping acting from $E$ into $E^{*}$, defined by the formula: $\partial_{f}(x):=\left\{x^{*} \in E^{*}:\left\langle y-x, x^{*}\right\rangle \leq f(y)-f(x)\right.$ for every $\left.y \in E\right\}, x \in E$. It easily follows by this definition that $\partial_{f}$ is a monotone mapping, i.e., for every $x_{1}, x_{2} \in E$ and for every $x_{1}^{*} \in \partial_{f}\left(x_{1}\right)$ and $x_{2}^{*} \in \partial_{f}\left(x_{2}\right)$ one has $\left\langle x_{1}-x_{2}, x_{1}^{*}-\right.$ $\left.x_{2}^{*}\right\rangle \geq 0$.

The following facts are well-known and may be found in [Ph].

(i) $\partial_{f}: E \rightarrow E^{*}$ is a norm-to- $w^{*}$ u.s.c.o. mapping with $\operatorname{Dom}\left(\partial_{f}\right)=E$;

(ii) $f$ is Gâteaux differentiable at $x_{0} \in E$ iff $\partial_{f}\left(x_{0}\right)$ is a singleton;

(iii) $f$ is Fréchet differentiable at $x_{0} \in E$ iff $\partial_{f}$ is single-valued and normto-norm u.s.c. at $x_{0}$.

In 1933 Mazur [Ma] proved that if $E$ is a separable Banach space, then $f$ is Gâteaux differentiable at the points of some residual subset of $E$ (in such a case we say that $f$ is generically Gâteaux differentiable). In 1968 Asplund [As] exhibited very general sufficient conditions under which $f$ is generically Gâteaux or Fréchet differentiable.

Let $d(\cdot, \cdot)$ be a metric in $E^{*}$. It is said that $d$ fragments $E^{*}$ (see [JR]) if for every bounded subset $A$ of $E^{*}$ and every $\varepsilon>0$ there exists some $w^{*}$-open subset $V$ of $E^{*}$ such that $V \cap A \neq \varnothing$ and $d$-diam $(V \cap A)<\varepsilon$. It is well-known that $E^{*}$ has Radon-Nikodym Property (RNP) iff $E^{*}$ is fragmented by the metric generated by the dual norm in $E^{*}$. Another important example is provided in the paper of Ribarska [Ri]: if the norm in $E$ is Gâteaux differentiable at each $x \in E, x \neq 0$, then $E$ is fragmented by some metric $d$.

The following theorem is well-known.

Theorem 6.5. Let $E$ be a Banach space with dual $E^{*}$ fragmented by some metric $d$ and $f: E \rightarrow \mathbf{R}$ be a continuous convex function. Then there exists a dense $G_{\delta}$-subset $D$ of $E$ at the points of which $f$ is Gâteaux differentiable. If the metric $d$ is generated by the dual norm in $E^{*}$ then at the points of $D f$ is Fréchet differentiable.

Proof. Since $\partial_{f}:(E,\|\cdot\|) \rightarrow\left(E^{*}, w^{*}\right)$ is u.s.c.o. there exists some minimal u.s.c.o. $F:(E,\|\cdot\|) \rightarrow\left(E^{*}, w^{*}\right)$ which is a selection of $\partial_{f}$. The following lemma gives us the possibility to apply Theorem 5.1.

Lemma 6.6. The mapping $F:(E,\|\cdot\|) \rightarrow\left(E^{*}, d\right)$ is fragmented by the metric $d$.

Proof. Consider the closed unit ball $B^{*}$ in $E^{*}$. Since $F$ is norm-to- $w^{*}$ u.s.c.o. the sets $F^{-1}\left(n B^{*}\right)$ are closed in $X$ for every $n \geq 1$. Take an arbitrary nonempty open set $U$ of $E$ and positive $\varepsilon$. Observe that $U \subset \bigcup_{n=1}^{\infty} F^{-1}\left(n B^{*}\right)$. By the Baire theorem $U_{1}:=U \cap \operatorname{Int} F^{-1}\left(n B^{*}\right) \neq \varnothing$ for some $n \geq 1$. Since $F$ is 
minimal we get $F\left(U_{1}\right) \subset n B^{*}$ (Proposition 2.1 (b)). On the other hand, $d$ fragments $E^{*}$, hence there exists a $w^{*}$-open set $V$ of $E^{*}$ such that $V \cap F\left(U_{1}\right) \neq \varnothing$ and $d$-diam $\left(V \cap F\left(U_{1}\right)\right)<\varepsilon$. Again by the minimality of $F$ (Proposition 2.1 (c)) one gets a nonempty open $U^{\prime} \subset U_{1}$ such that $F\left(U^{\prime}\right) \subset V$. Therefore $d$-diam $\left(F\left(U^{\prime}\right)\right)<d$-diam $\left(V \cap F\left(U_{1}\right)\right)<\varepsilon$. The proof of the lemma is completed.

Now, let us go back to the proof of Theorem 6.5. By Theorem 5.1 there exists a dense $G_{\delta}$-subset $D$ of $E$ at the points of which $F$ is single-valued and norm-to- $d$ u.s.c. We will show further that

(i) $\partial_{f}(x)=F(x)$ for every $x \in D$ (i.e., $F$ is Gâteaux differentiable at the points of $D$ );

(ii) if the metric $d$ is generated by the dual norm in $E^{*}$ then $\partial_{f}$ is singlevalued and norm-to-norm u.s.c. at any $x \in D$ (this would imply that $f$ is Fréchet differentiable at the points of $D$ ).

We will prove (ii) now. Let $x_{0} \in D$ and $\varepsilon>0$. Then $F\left(x_{0}\right)=\left\{x_{0}^{*}\right\}$ for some $x_{0}^{*} \in E^{*}$ and $x_{0}^{*} \in \partial_{f}\left(x_{0}\right)$. Since $F$ is norm-to-norm u.s.c. at $x_{0}$ there exists some open $U$ of $X$ such that $x_{0} \in U$ and $F(U) \subset x_{0}^{*}+\varepsilon B^{*}$. It suffices to show that $\partial_{f}(U) \subset x_{0}^{*}+\varepsilon B^{*}$. Suppose this is not the case and take some $x_{1}^{*} \in \partial_{f}\left(x_{1}\right) \backslash\left\{x_{0}^{*}+\varepsilon B^{*}\right\}$ where $x_{1} \in U$. Then there exists $h \in E,\|h\|=1$, which strongly separates $x_{1}^{*}$ from the close convex ball $x_{0}^{*}+\varepsilon B^{*}$, i.e., for some $\delta>0$ the $w^{*}$-open set $H_{\delta}:=\left\{x^{*} \in E^{*}:\left\langle h, x^{*}\right\rangle>\left\langle h, x_{1}^{*}\right\rangle-\delta\right\}$ does not intersect $x_{0}^{*}+\varepsilon B^{*}$.

Consider, for $t>0$, the point $x(t):=x_{1}+t h$. By the monotonicity of $\partial_{f}$ we have

$$
0 \leq\left\langle x(t)-x_{1}, x^{*}-x_{1}^{*}\right\rangle=t\left\langle h, x^{*}-x_{1}^{*}\right\rangle \text { for every } x^{*} \in \partial_{f}(x(t)) .
$$

This means that $\partial_{f}(x(t)) \subset H_{\delta}$ for every $t>0$. However, when $t$ is small enough $x(t) \in U$ and hence $F(x(t)) \subset x_{0}^{*}+\epsilon B^{*}$. This is a contradiction since $F$ is a selection of $\partial_{f}$. The proof of (ii) is completed. The proof of (i) is even simpler.

In the next theorem the Banach space $E$ is identified with its natural embedding in its second dual $E^{* *}$.

Theorem 6.7. Let $E$ be a separable Banach space and $f: E^{*} \rightarrow \mathbf{R}$ be a continuous convex function such that the set $A:=\left\{x^{*} \in E^{*}: \partial_{f}\left(x^{*}\right) \cap E \neq \varnothing\right\}$ is residual in the norm topology in $E^{*}$. Then $f$ is Fréchet differentiable on a dense $G_{\delta}$-subset $A^{\prime}$ of $E^{*}$ and $\partial_{f}\left(x^{*}\right) \in E$ for every $x^{*} \in A^{\prime}$.

Proof. Consider the mapping $F: A \rightarrow E$ defined by $F\left(x^{*}\right):=\partial_{f}\left(x^{*}\right) \cap E$, $x^{*} \in A$. Evidently $\operatorname{Dom}(F)=A$. On the other hand, since $\partial_{f}$ has a closed graph, the mapping $F$ has a closed graph too. Hence, by Theorem 4.16 there exist a residual subset $A^{\prime}$ of $A$ and a continuous single-valued mapping $h$ : $A^{\prime} \rightarrow E$ which is a selection of $F$. Hence $A^{\prime}$ is residual in $E^{*}$. Arguments similar to the used in the proof of (ii) from Theorem 6.5 show that for every $x^{*} \in A^{\prime} \partial_{f}\left(x^{*}\right)=h\left(x^{*}\right)$ and $\partial_{f}$ is norm-to-norm u.s.c. at $x^{*}$. This means that $f$ is Fréchet differentiable at the points of $A^{\prime}$. 
Put, further, $B P:=\left\{x^{*} \in E^{*}: x^{*}\right.$ attains its maximum on the unit ball $\left.B\right\}$. According to the famous Bishop-Phelps theorem [BPh], the set $B P$ is dense in the norm topology in $E^{*}$.

Corollary 6.8. Let $E$ be a separable Banach space such that the Bishop-Phelps set $B P$ is residual in $E^{*}$. Then the norm in $E^{*}$ is Fréchet differentiable at the points of some residual subset $A^{\prime}$ of $E^{*}$ and for every $x^{*} \in A^{\prime} \partial_{\|\cdot\| \|}\left(x^{*}\right) \in B$. In particular, for every $x^{*} \in A^{\prime}$ the maximization problem $\left(B, x^{*}\right)$ is Tikhonov well-posed.

Proof. Let $B^{* *}$ be the closed unit ball in $E^{* *}$. It is well known that $\partial_{\|\cdot\|}\left(x^{*}\right)=$ $\left\{x^{* *} \in B^{* *}:\left\langle x^{*}, x^{* *}\right\rangle=\left\|x^{*}\right\|\right\}$ and that $\partial_{\|\cdot\|}\left(x^{*}\right) \cap E=\partial_{\|\cdot\|}\left(x^{*}\right) \cap B \neq \varnothing$ iff $x^{*}$ attains its maximum on $B$. Therefore, $B P=\left\{x^{*} \in E^{*}: \partial_{\|\cdot\|}\left(x^{*}\right) \cap E \neq \varnothing\right\}$. It remains to apply Theorem 6.7.

Remark 6.9. As shown in [KG, Theorem 3.5] the last two statements are valid for all Banach spaces which admit an equivalent locally uniformly rotund norm.

(d) Metric projections and antiprojections in Banach spaces. Let again $E$ be a real Banach space with norm $\|\cdot\|$. Denote by $S$ the unit sphere of $E$, i.e., the set $\{x \in E:\|x\|=1\}$. Recall that the norm $\|\cdot\|$ in $E$ is locally uniformly rotund if for every $x_{0}, x_{n} \in S$ such that $(1 / 2)\left\|x_{0}+x_{n}\right\| \rightarrow 1$, it follows that $x_{n} \rightarrow x_{0} .\|\cdot\|$ is strictly convex if $S$ does not contain line segments.

Let $A \subset E$ be a nonempty subset of $E$. The metric projection generated by $A$ is the multivalued mapping $P_{A}: E \rightarrow A$ defined by $P_{A}(x):=\{y \in A$ : $\left.\|x-y\|=\inf \left\{\left\|x-y^{\prime}\right\|: y^{\prime} \in A\right\}\right\}$. The conjecture that the set $\left\{x \in E: P_{A}(x)=\right.$ $\varnothing$ or $P_{A}(x)$ is a singleton $\}$ is residual in $E$ provided the norm in $E$ is strongly convex was stated by Steckin [St]. Up to now there are partial positive answers to this question (see [FaZh, Ko1, Ko2, L, St, Za, Zh]).

Analogously, if $A$ is a nonempty and bounded subset of $E$ one can consider the metric antiprojection mapping $Q_{A}: E \rightarrow A$ defined by $Q_{A}(x):=\{y \in A$ : $\left.\|x-y\|=\sup \left\{\left\|x-y^{\prime}\right\|: y^{\prime} \in A\right\}\right\}$. From the above point of view the metric antiprojection is investigated in [PaKa, $\mathrm{Zh}$ ].

We prove here the original result of [St] about metric projections in a slightly stronger form containing Theorem 1.8 from $[\mathrm{Zh}]$.

Theorem 6.10. Let $E$ have a locally uniformly rotund norm and $A$ be closed (resp. closed and bounded). Then there exists a dense $G_{\delta}$-subset $E_{1}$ of $E$ at the points of which $P_{A}$ (resp. $Q_{A}$ ) is no more than single-valued and u.s.c. Moreover, if $\operatorname{Dom}\left(P_{A}\right)\left(\right.$ resp. $\left.\operatorname{Dom}\left(Q_{A}\right)\right)$ is dense in $E$ then $E_{1} \subset \operatorname{Dom}\left(P_{A}\right)$ (resp. $\left.E_{1} \subset \operatorname{Dom}\left(Q_{A}\right)\right)$.

Proof. We prove the theorem only for metric projections. The case of antiprojections is analogous.

Let $X:=\operatorname{ClDom}\left(P_{A}\right)$. The set $E \backslash X$ is open and if it is nonempty then obviously for every $x \in E \backslash X P_{A}(x)=\varnothing$ and $P_{A}$ is u.s.c. at $x$. So consider $Y:=\operatorname{Int} C l \operatorname{Dom}(F)$. The set $X \backslash Y$ is nowhere dense in $E$. Hence, if $Y=\varnothing$, we are done. On the other hand, if $Y \neq \varnothing$ and one proves that there is a dense $G_{\delta}$-subset $X_{1}$ of $X$ such that $P_{A}$ is single-valued and u.s.c. in $X$ at each point of $X_{1}$, then one can easily get the conclusion of the theorem.

So, consider $P_{A}$ only in $X$. Observe that $X$ is a complete metric space, hence a Baire space, and moreover $\operatorname{Dom}\left(P_{A}\right)$ is dense in $X$. On the other hand, 
by the fact that $A$ is closed one can easily see that $\operatorname{Gr}\left(P_{A}\right)$ is a closed subset of $X \times A$. Further, let $x_{0} \in X$ and $P_{A}\left(x_{0}\right) \neq \varnothing$. By Lemma 1.7 from [Zh] for every $y \in P_{A}\left(x_{0}\right)$ the metric projection $P_{A}$ is upper semicontinuous and single-valued at each point $x^{\prime}$ from the interior of the line segment $\left[x_{0}, y\right]$. Since for every such $x^{\prime} P_{A}\left(x^{\prime}\right)=y$ we easily get that Int $P_{A}^{\#}(V)$ is dense in $P_{A}^{-1}(V)$ for every $V$ open in $A$. Hence, by Theorem 5.3 , there exists a dense $G_{\delta}$-subset $X_{1}$ of $X$ such that $X_{1} \subset \operatorname{Dom}\left(P_{A}\right)$ and $P_{A}$ is single-valued and u.s.c. at the points of $X_{1}$. The proof is completed.

\section{REFERENCES}

[AL] J. M. Aarts and D. Lutzer, Completeness properties designed for recognizing Baire spaces, Dissertationes Math. (Rozprawy Mat.) 116 (1974), 1-48.

[As] E. Asplund, Fréchet differentiability of convex functions, Acta Math. 121 (1968), 31-47.

[BPh] E. Bishop and R. R. Phelps, A proof that every Banach spaces is subreflexive, Bull. Amer. Math. Soc. 67 (1961), 97-98.

[BLL] D. Burke, D. Lutzer and S. Levi, Functional characterization of certain p-spaces, Topology Appl. 20 (1985), 161-165.

[CČN] J. Chaber, M. M. Čoban, and K. Nagami, On monotonic generalizations of Moore spaces, Čech-complete spaces and p-spaces, Fund. Math. 84 (1974), 107-119.

[Chr] J. P. R. Christensen, Theorems of I. Namioka and R. E. Jonson type for upper semicontinuous and compact-valued set-valued maps, Proc. Amer. Math. Soc. 86 (1982), 649655.

[ChrK] J. P. R. Christensen and P. S. Kenderov, Dense strong continuity of mappings and the Radon-Nikodym property, Math. Scand. 54 (1984), 70-78.

[ČK1] M. M. Čoban and P. S. Kenderov, Dense Gâteaux differentiability of the sup-norm in $C(T)$ and the topological properties of $T, C$. R. Acad. Bulgare. Sci. 38 (1985), 16031604 .

[ČK2] Generic Gâteaux differentiability of convex functionals in $C(T)$ and the topological properties of $T$, Math. and Education in Math., Proc. 15th Spring Conf. of the Union of Bulgare Math., 1986, pp. 141-149.

[ČKR1] M. M. Čoban, P. S. Kenderov and J. P. Revalski, Generic well-posedness of optimization problems in topological spaces, C. R. Acad. Bulgare Sci. 42(1989), 11-14.

[ČKR2] - Generic well-posedness of optimization problems in topological spaces, Mathematika 36 (1989), 301-324.

[DK] F. Deutsch and P. S. Kenderov, Continuous selections and approximate selection for setvalued mappings and applications to metric projections, SIAM J. Math. Anal. 14 (1983), 185-194.

[En] R. Engelking, General topology, PWN, Warsaw, 1977.

[FaZh] M. Fabian and N. V. Zhivkov, A characterization of Asplund spaces with the help of local $\varepsilon$-supports of Ekeland and Lebourg, C. R. Acad. Bulgare. Sci. 38 (1985), 671-674.

[Fro] Z. Frolik, Generalizations of the $G_{\delta}$-property of complete metric spaces, Czechoslovak. Math. J. 10 (1960), 359-379.

[FV] M. Furi and A. Vignoli, About well-posed minimization problems for functionals in metric spaces, J. Optim. Theory Appl. 5 (1970), 225-290.

[HJT] R. W. Hansell, J. E. Jayne and M. Talagrand, First class selector for weakly upper semicontinuous multivalued maps in Banach spaces, J. Reine Angew. Math. 361 (1985), 201220.

[HS] H. Herrlich and C. E. Strecker, H-closed spaces and reflective subcategories, Math. Ann. 177 (1968), 302-309.

[JR] J. E. Jayne and C. A. Rogers, Borel selectors for upper semi-continuous set-valued maps, Acta Math. 155 (1985), 41-79. 
[K1] P. S. Kenderov, Dense strong continuity of pointwise continuous mappings, Pacific J. Math. 88 (1980), 111-130.

[K2] Continuity-like properties of multivalued mappings, Serdica 9 (1983), 149-160.

[KG] P. S. Kenderov and J. R. Giles, On the structure of Banach spaces with Mazur's intersection property, Math. Ann. 291 (1991), 463-473.

[Kol] S. V. Konyagin, Approximative properties of subsets in Banach spaces, Dokl. Akad. Nauk SSSR 239 (1978), 261-264.

[Ko2] - Approximative properties of closed subsets in Banach spaces and characterizations of strongly convex spaces, Dokl. Akad. Nauk SSSR 251 (1980), 276-279.

[L] Ka-S. Lau, Almost Chebyshev subsets in reflexive Banach spaces, Indiana Univ. Math. J. 27 (1978), 791-795.

[Ma] S. Mazur, Über konvexe Mengen in linearen normierten Räumen, Studia Math. 4 (1933), 70-84.

[M1] E. Michael, Continuous selections I. Ann. of Math. (2) 63 (1956), 562-590.

[M2] , A theorem on semi-continuous set-valued functions, Duke Math. J. 26 (1959), 647-652.

[M3] - Complete spaces and tri-quotient maps, Illinois J.Math. 21 (1977), 716-733.

[M4] Almost complete spaces, hypercomplete spaces and related mapping theorems, Topology Appl. 41 (1991), 113-130.

[Na] I. Namioka, Separate continuity and joint continuity, Pacific J. Math. 51 (1974), 515-531.

[PaKa] B. B. Panda and O. P. Kapoor, On farthest points of sets, J. Math. Anal. Appl. 62 (1978), 345-353.

[Pas] B. A. Pasynkov, Zero-dimensional open mappings raising the dimension, Uspehi Mat. Nauk 18 (1963), 183-190. (Russian)

[Ph] R. R. Phelps, Convex functions, monotone operators and differentiability, Lecture Notes in Math., vol. 1364, Springer-Verlag, Berlin, 1989.

[Ri] N. Ribarska, The dual of a Gâteaux smooth Banach space is weak star fragmentable, Proc. Amer. Math. Soc. (to appear).

[Ro] H. P. Rosenthal, The heredity problem for weakly compactly generated Banach spaces, Compositio Math. 28 (1974), 83-111.

[St] S. B. Stečkin, Approximative properties of Banach spaces subsets, Rev. Roumaine Math. Pures Appl. 8 (1963), 5-8.

[S] C. Stegall, Topological spaces with dense subspaces that are homeomorphic to complete metric spaces and the classification of $C(K)$ Banach spaces, Mathematika 34 (1987), 101-107.

[T] A. N. Tikhonov, On the stability of the functional optimization problem, U.S.S.R. Comput. Math. and Math. Phys. 6 (1966), 631-634.

[Za] L. Zajčik, On the points of multivaluedness of metric projections in separable Banach spaces, Comment. Math. Univ. Carolin. 19 (1978), 513-523.

[Zh] N. V. Zhivkov, Continuity and non-multivaluedness properties of metric projections and antiprojections, Serdica 8 (1982), 378-385.

Krasnodonskaya 72, KV. 119, 278000 TiRaspol, Moldova

Institute of Mathematics, Bulgarian Academy of Sciences, Acad. G. Bonchev Street, BLocK 8, 1113 Sofia, BUlgaria

E-mail address: kenderov@bgearn.bitnet

E-mail address: revalski@bgearn.bitnet 